\title{
Spillover Effects of the Hospital Readmissions Reduction Program on Radical Cystectomy Readmissions
}

\author{
Matthew S. Lee ${ }^{1}$, Brent K Hollenbeck ${ }^{1}$, Mary K. Oerline ${ }^{1}$, Ted A. Skolarus ${ }^{1}$, Bruce L. \\ Jacobs $^{3}$, Rita Jen ${ }^{1}$, Amy N. Luckenbaugh ${ }^{1}$, Vahakn Shahinian ${ }^{1}$, Tudor Borza ${ }^{2}$ \\ Matthew S. Lee: lematthe@med.umich.edu; Brent K Hollenbeck: bhollen@med.umich.edu; Mary K. Oerline: \\ mare@med.umich.edu; Ted A. Skolarus: tskolar@med.umich.edu; Bruce L. Jacobs: jacobsbl2@upmc.edu; Rita Jen: \\ rjen@med.umich.edu; Amy N. Luckenbaugh: amy.n.luckenbaugh@vanderbilt.edu; Vahakn Shahinian: \\ vahakn@med.umich.edu; Tudor Borza: borza@urology.wisc.edu \\ ${ }^{1}$ Dow Division for Health Services Research, Department of Urology, University of Michigan, Ann \\ Arbor, MI \\ ${ }^{2}$ Department of Urology, University of Wisconsin, Madison, WI \\ ${ }^{3}$ Department of Urology, University of Pittsburgh Medical Center, Pittsburgh, PA
}

\section{Abstract}

Introduction and Objective: Readmission rates after radical cystectomy are among the highest of any surgery. The Hospital Readmissions Reduction Program (HRRP) penalizes hospitals with excess readmissions for certain targeted conditions, including total hip and knee arthroplasty. We examined whether changes made by hospitals in response to the HRRP had spillover effects on radical cystectomy readmissions.

Methods: We used a 20\% sample of Medicare data to identify patients undergoing cystectomy from 2010 to 2014 and measured 30-day adjusted cystectomy readmission rates. To determine the effect of the HRRP, we calculated adjusted readmission rates following total hip or knee arthroplasty and stratified hospitals into quartiles (most improved, middle quartiles, least improved) based on their improvement in reducing those targeted readmissions. Multivariable logistic regression was used to identify factors associated with spillover effects from the targeted joint surgery to cystectomy.

Results: We identified 2,394 patients undergoing radical cystectomy. Of these, 606 were treated at hospitals in the "most improved" quartile and 522 in the "least improved." Patients undergoing cystectomy were similar in age, comorbidity, and SEC independent of hospital performance quartile. The readmission rate following cystectomy was $26 \%$ in the most improved quartile and $24 \%$ in the least improved. No spillover effect was identified between readmission reduction after major joint surgery and radical cystectomy (adjusted OR 0.90, $\mathrm{p}=0.42$ ).

Conclusions: Hospitals that succeeded in reducing readmissions following major joint surgery targeted by the HRRP did not have similar reductions in readmissions following radical

Corresponding author: Tudor Borza MD, MS, Department of Urology, University of Wisconsin School of Medicine and Public Health, 1685 Highland Ave, Madison, WI, 53705, borza@ urology.wisc.edu, Office \# 608-262-5440, Fax \# 608-262-6453.

Disclosures: The authors have no conflicts of interest related to this work.

Disclaimer: The contents do not represent the views of the United States Government. 
cystectomy. This lack of spillover effect suggests that each surgical condition may require tailored interventions to prevent readmissions.

\section{Keywords}

bladder; cancer; readmissions

\section{Introduction}

Radical cystectomy is a high risk procedure with complications occurring in approximately $50 \%$ of patients. ${ }^{1,2}$ While complications, in part, reflect the complexity of the procedure itself, they are also a consequence of the underlying patient population, which is older and has multiple coexisting health issues. Not surprisingly, approximately $25 \%$ of patients are readmitted after cystectomy 2,3 , one of the highest rates among all surgical procedures. Despite a variety of measures aimed at improving perioperative care ${ }^{4-6}$, readmissions have remained stable over the past decade ${ }^{7}$. Furthermore, these readmissions are expensive ${ }^{8,9}$ and associated with an increased risk of dying ${ }^{1}$.

Reducing unplanned readmissions, many of which are thought to be avoidable ${ }^{10,11}$, has become a priority for the Centers for Medicare and Medicaid Services (CMS) as evidenced by the implementation and expansion of the Hospital Readmissions Reduction Program (HRRP). This policy aims to constrain healthcare spending by penalizing hospitals for higher than anticipated readmissions within 30 days for select medical and surgical conditions ${ }^{12}$. Indeed, early analyses suggest that the policy is achieving its desired effect for the targeted conditions ${ }^{13,14}$. For surgical conditions, the policy is currently limited to major joint replacement (i.e. total hip and knee arthroplasty) and coronary artery bypass grafting. However, in light of the early success, many expect the scope of the policy to broaden to other surgical discharges with high readmission rates, such as radical cystectomy. To avoid financial penalties from excess readmissions, hospitals have implemented broad-based efforts, such as enhanced patient navigation and care coordination of post-discharge followup ${ }^{15,16}$, which have the potential to positively impact all surgical discharges. In contrast, some hospitals have expanded the scope of their quality improvement efforts with a specific focus on only the targeted procedures, such as enhanced Methicillin-Resistant Staphylococcus Aureus (MRSA) decolonization protocols ${ }^{17}$ or intensive physical therapy for patients undergoing major joint surgery ${ }^{18}$. Given their limited scope, implementation of these interventions may not have an impact on readmissions in other surgical populations.

Readmission reduction is a clear strategic priority for CMS to contain growing healthcare costs and hospitals appear to be responsive to the threat of penalties from the HRRP ${ }^{14,19}$. Insofar as strategies implemented by hospitals to reduce readmissions among procedures targeted by the HRRP are broad-based, there is the potential for their effect to spill over to other patient populations, such as those following radical cystectomy. For this reason, we assessed readmissions following radical cystectomy, stratified by a hospital's ability to reduce surgical readmissions targeted by the HRRP. Our hypothesis was that hospitals that were effective in reducing readmissions after major joint surgery may have implemented broad-based initiatives with effects on non-targeted surgeries. 


\section{METHODS}

Study Population-Using a 20\% national Medicare sample, we performed a retrospective cohort study of fee-for-service Medicare beneficiaries between 2010 and 2014-the period during which CMS began measuring and penalizing hospitals as part of the HRRP. We identified patients undergoing cystectomy over this time period using International Classification of Diseases, $9^{\text {th }}$ Revision, procedure codes (57.7, 57.71, 57.79). The cohort was further restricted to those patients carrying a diagnosis of bladder cancer (188.x, 233.7) to ensure only patients undergoing radical cystectomy were included. For the purpose of risk adjustment and to ensure reliability of assessing readmission, patients were included if they were continually enrolled in Medicare Parts A and B for at least one year prior to cystectomy through 30-days after discharge. Due to absence of complete claims, patients enrolled in Medicare Advantage plans were excluded. To be consistent with the HRRP requirements for major joint surgery, we restricted our cohort to patients undergoing cystectomy at hospitals performing 5 or more total hip and total knee arthroplasties annually (translates to $\geq 25$ total hip and knee arthroplasties in a 100\% Medicare cohort), as only hospitals performing at least 25 joint surgeries annually are subject to HRRP penalties. In our primary analysis, hospitals performing at least 1 radical cystectomy were included (translates to at least 5 cystectomies in the $100 \%$ Medicare cohort). Therefore, only patients undergoing radical cystectomy at hospitals performing major joint surgery were included in our analysis.

For each hospital, we calculated annual risk-adjusted rates of readmission for total hip or total knee arthroplasty, using patient level logistic regression and adjusting for patient age, hierarchical condition categories, and type of procedure (total hip or total knee arthroplasty). The risk adjusted readmission rate from 2010 for major joint surgery was then subtracted from the adjust readmission rate from 2014 to calculate change in readmissions rates. Hospitals were identified by their hospital ID (Medicare Provider Number). We then stratified hospitals into quartiles (most improved, middle quartiles, least improved) by their ability to reduce readmissions after the targeted major joint surgery. We hypothesized that hospitals with the greatest improvement in readmissions, as opposed to the lowest overall readmission rates, after the targeted procedures were most likely to have implemented new, broad-based initiatives to reduce readmissions and have the greatest potential for spillover effects to the cystectomy population.

Our primary outcome was readmission occurring within 30 days following discharge for patients undergoing radical cystectomy. The 30-day window was chosen to be consistent with how CMS assesses readmissions for the HRRP ${ }^{12}$.

Assessing spillover effects of the HRRP-We first compared patient and hospital characteristics among cystectomy patients according to hospitals' HRRP performance quartile. Statistical inference was made using t-tests and chi square tests for continuous and categorical data, respectively. Multivariable logistic regression - adjusting for patient (age, race, comorbidity, socioeconomic class, residential area) and hospital variables (size, urban vs. rural location, teaching and for-profit status) - was used to calculate adjusted readmission rates following cystectomy. Comorbidity was determined using patient claims 
data for the 12-month window prior to diagnosis using established methods (Hierarchical Condition Categories - HCC) ${ }^{20}$. Socioeconomic class was estimated using a composite measure developed at the five-digit zip code level as described by Diez-Roux ${ }^{21}$.

Lastly, we examined the extent to which readmissions within a hospital for cystectomy and major joint surgery correlated with one another. To do this, we used generalized estimating equations to model readmission after cystectomy using a multi-level framework (i.e., patients nested within hospitals). Therefore, patient level readmissions were aggregated to the hospital level to calculate hospital level readmission rates. The model was adjusted for several patient-level covariates, as mentioned above. Using data from the American Hospital Association, the model was further adjusted for hospital characteristics, including cystectomy volume, degree of urbanization, bed count, and for-profit status. Robust standard errors were used to account for clustering of patients within hospitals. We then used Pearson correlation to calculate the correlation between readmissions following cystectomy and major joint surgery. Odds ratios were calculated to predict the risk of readmission based on hospital quartile (most improved, middle quartiles, least improved).

As a secondary analysis, we calculated the Pearson correlation coefficient by plotting risk adjusted readmission rates following cystectomy against readmission rates following major joint surgery. The cohort was further restricted to hospitals that performed $\geq 5$ cystectomies per year (translates to $\geq 25$ cystectomies per year). The purpose of this analysis was to determine if the hospital's absolute readmission rates (as opposed to difference from 2010 to 2014) correlated between joint surgery and cystectomy, therefore, adjusted readmission rates were plotted against each other, not change in readmission rates.

All analyses were carried out using SAS 9.4 (Cary, NC). All tests were two-tailed and the probability of Type 1 error was set at 0.05 . The study protocol was judged to be exempt by the institutional review board of the University of Michigan.

\section{Results}

We identified 2,394 patients who underwent radical cystectomy at 570 hospitals. Patients were similar in age, comorbidity (HCCs), and socioeconomic class independent of how their hospital performed with respect to readmission after major joint surgery. There were, however, some clinically relevant differences, as shown in Table 1 . Patients in very large cities ( $>1$ million people) tended to receive care at hospitals in the least improved quartile. Hispanic and Asian patients also tended to receive their care at hospitals in the least improved quartile. Larger hospitals (>250 beds) and teaching institutions were more likely to be in the most improved quartile.

The median adjusted readmission rate following radical cystectomy was $26 \%$ in the most improved quartile; $25 \%$ and $26 \%$ for the second and third-most improved quartiles, respectively (middle quartiles); and $24 \%$ for the least improved quartile hospitals. These differences were not statistically significant $(\mathrm{p}=0.42)$. Figure 1 illustrates annual adjusted readmission rates after radical cystectomy from 2010 to 2014 stratified by hospital performance quartile. There was no consistent trend identified. Compared to hospitals in the 
most improved quartile, the odds of avoiding readmission to the least improved quartile hospitals was 0.90 ( $\mathrm{p}$ value $=0.42$ ). The odds ratios of avoiding readmission to the secondand third-most improved quartile hospitals were; 0.93 (p-value 0.59) and 0.95 (p-value 0.75 ), respectively (results not shown).

The relationship between readmission following radical cystectomy and major joint surgery is further illustrated in Figure 2. Here, we further restricted our cohort to only analyze hospitals that performed $\geq 5$ hip or knee arthroplasties and $\geq 5$ cystectomies per year. 1585 patients underwent radical cystectomy at 138 hospitals. The Pearson correlation coefficient was 0.02 ( $\mathrm{p}$ value $=0.80$ ), demonstrating no identifiable trend in readmissions after radical cystectomy and major joint surgery.

\section{Discussion}

Improvement in 30-day readmission rates following major joint surgery targeted by the HRRP did not spill over to readmission for patients undergoing radical cystectomy. We found no relationship between 30-day readmission following radical cystectomy and a hospital's ability to reduce readmissions following major joint surgery targeted by the HRRP. From a policy perspective, interventions intended to reduce post-surgical readmission would ideally translate across all surgical discharges; however, this may not be the case.

Successful interventions to reduce readmissions following major joint surgery may be too focused to have an effect on other surgical conditions. Radical cystectomy patients face different peri- and post-operative complications compared to those undergoing major joint surgery. For example, the most common reasons for 30-day readmission after major joint surgery include wound infection and hardware malfunction ${ }^{22-24}$, whereas, for radical cystectomy the most common diagnoses at readmission include ileus, pyelonephritis/urinary tract infection, dehydration, difficulty managing urostomy and deep venous thrombosis $1-3,25,26$. Therefore, potential interventions to reduce readmissions after major joint surgery - such as MRSA decolonization protocols, which decreased the incidence of deep surgical infections by $13 \%$, or use of pre-operative physical therapy to improve mobility-may not help reduce cystectomy readmissions $17[10,29]$. Similarly, addressing intraoperative risks factors associated with readmission after major joint surgery, such as procedure and tourniquet time or peri-operative blood transfusion are unlikely to have an effect outside of major joint surgery 24,27 .

There is wide variation in readmission rates for different surgeries. A retrospective analysis of 22,559 patients at a single academic medical center across eight different general surgery subspecialties identified wide variation in the incidence of complications and readmissions, with 30-day readmission rates ranging from $2.1 \%$ following breast, melanoma, or endocrine surgery to as high as $24.8 \%$ following transplant surgery. The authors concluded that $82.8 \%$ of the variability in 30-day readmission rates was attributable to non-modifiable factors at the patient level, $14.5 \%$ attributable to surgical sub-specialty, and $2.8 \%$ attributable to individual surgeon ${ }^{28}$. Therefore, a certain percentage of readmissions may not be preventable. This seems particularly true following cystectomy, where improvements in perioperative care have not translated to reductions in readmissions. Enhanced recovery 
pathways for radical cystectomy have decreased length of stay and costs, however, readmission rates remain unchanged ${ }^{4,29}$. In another review of 1163 patients who underwent cystectomy across the United States, the authors concluded that only $26 \%$ of cystectomy readmissions may have been modifiable (factors controlled by the surgeon, e.g. surgical technique or judicious antibiotic administration) ${ }^{3}$. This suggests that a substantial percentage of radical cystectomy readmissions may not have modifiable risk factors for prevention.

Discharge planning has been associated with reductions in readmission in a variety of contexts. Higher quality discharge planning was associated with readmission reduction for congestive heart failure, total hip and knee arthroplasty, and pneumonia ${ }^{15}$. Those authors were surprised to find that discharge planning had an effect on major joint surgery readmissions since discharge planning is unlikely to reduce infectious complications, the most common cause for readmission. Furthermore, since joint surgery is elective, the authors presumed that modifiable risk factors would have already been addressed. Given the unexpected result, it is possible that quality discharge planning could also reduce cystectomy readmissions. However, effective discharge planning, likely needs to be implemented with the specific population in mind to have an effect. For example, Krishnan et al, used a systems engineering approach to identify that patients after radical cystectomy are likely to exhibit concerning symptoms at post-discharge day 4 or $5{ }^{30}$. A focus on outpatient care coordination around this critical time (e.g. ensuring medication compliance and adequate hydration) could potentially decrease readmission rates.

The findings of our study should be interpreted in the context of several limitations. First, we limited our analysis to Medicare beneficiaries age 66 years and older. However, as bladder cancer usually affects the elderly, this has minimal implications for the generalizability of our findings. Second, in our study, quality was defined as the ability to reduce readmissions after major joint surgery in response to the HRRP. Improvement in readmission reduction may not necessarily indicate quality, as hospitals with lower baseline readmission rates, may be unable to achieve further reductions. Indeed, the hospitals with lowest readmission rates are likely in the middle quartiles. However, the purpose of our study was to evaluate the effect of the HRRP and to identify beneficial, unintended effects of the program, not to identify the hospitals with the lowest readmission rates. Therefore, it is reasonable to hypothesize that a spillover effect might exist if initiatives implemented to reduce readmissions after major joint surgery were broad-based and that if spillover effects did exist, they would most likely be identified in the hospitals with the most improvement in readmission reduction. As discussed above, no effect may have been identified due to lack of modifiable risk factors and differences in the complexity of the surgical populations or if the procedures implemented for joint surgery patients are still siloed within the orthopedic surgery departments and have not been implemented hospital-wide.

\section{Conclusion}

A hospital's ability to reduce readmissions following surgery targeted by the HRRP was not associated with similar reductions in radical cystectomy readmission rates. This suggests that successful interventions were likely implemented only in the targeted populations or 
that unique, tailored interventions will be necessary to prevent readmissions given the heterogeneity in patient populations and post-operative complications across surgical discharges.

\section{Acknowledgments}

Funding: This work was supported by the National Institute on Aging (R01 AG048071) and the National Cancer Institute (T32 CA180984).

\section{References:}

1. Gore JL, Lai J, Gilbert SM, Urologic Diseases in America P. Readmissions in the postoperative period following urinary diversion. World journal of urology. 2011;29(1):79-84. [PubMed: 21104414]

2. Stimson CJ, Chang SS, Barocas DA, et al. Early and late perioperative outcomes following radical cystectomy: 90-day readmissions, morbidity and mortality in a contemporary series. The Journal of urology. 2010;184(4):1296-1300. [PubMed: 20723939]

3. James AC, Izard JP, Holt SK, et al. Root Causes and Modifiability of 30-Day Hospital Readmissions after Radical Cystectomy for Bladder Cancer. The Journal of urology. 2016;195(4P1):894-899. [PubMed: 26555956]

4. Tyson MD, Chang SS. Enhanced Recovery Pathways Versus Standard Care After Cystectomy: A Meta-analysis of the Effect on Perioperative Outcomes. European urology. 2016.

5. Daneshmand S, Ahmadi H, Schuckman AK, et al. Enhanced recovery protocol after radical cystectomy for bladder cancer. The Journal of urology. 2014;192(1):50-55. [PubMed: 24518775]

6. Kauf TL, Svatek RS, Amiel G, et al. Alvimopan, a peripherally acting mu-opioid receptor antagonist, is associated with reduced costs after radical cystectomy: economic analysis of a phase 4 randomized, controlled trial. The Journal of urology. 2014;191(6):1721-1727. [PubMed: 24342144]

7. Jacobs BL, Zhang Y, Tan HJ, Ye Z, Skolarus TA, Hollenbeck BK. Hospitalization trends after prostate and bladder surgery: implications of potential payment reforms. The Journal of urology. 2013;189(1):59-65. [PubMed: 23164391]

8. Konety BR, Allareddy V. Influence of post-cystectomy complications on cost and subsequent outcome. The Journal of urology. 2007;177(1):280-287; [PubMed: 17162064]

9. Skolarus TA, Jacobs BL, Schroeck FR, et al. Understanding hospital readmission intensity after radical cystectomy. The Journal of urology. 2015;193(5):1500-1506. [PubMed: 25451833]

10. Feigenbaum PNE, Trowbridge L, Teplitsky S, Barnes CA, et al. Factors contributing to all-cause 30-day readmissions: a structured case series across 18 hospitals. Med Care. 2012;50:599-605. [PubMed: 22354212]

11. Vest JRGL, Oxford BA, Gonzalez MI, Slawson KM. Determinants of preventable readmissions in the United States: a systematic review. Implement Sci. 2010;5(88).

12. Services CfMM. Rules and Regulations. Federal Register. 2014;79(192).

13. Mehtsun WT, Papanicolas I, Zheng J, Orav EJ, Lillemoe KD, Jha AK. National Trends in Readmission Following Inpatient Surgery in the Hospital Readmissions Reduction Program Era. Ann Surg. 2017.

14. Borza T, Oerline MK, Skolarus TA, et al. Association of the Hospital Readmissions Reduction Program With Surgical Readmissions. JAMA Surg. 2017.

15. Henke RM, Karaca Z, Jackson P, Marder WD, Wong HS. Discharge Planning and Hospital Readmissions. Med Care Res Rev. 2016.

16. Goncalves-Bradley DC, Lannin NA, Clemson LM, Cameron ID, Shepperd S. Discharge planning from hospital. Cochrane Database Syst Rev. 2016(1):

17. Hadley S, Immerman I, Hutzler L, Slover J, Bosco J. Staphylococcus aureus Decolonization Protocol Decreases Surgical Site Infections for Total Joint Replacement. Arthritis. 2010;2010:924518. [PubMed: 22046511] 
18. Alem N, Rinehart J, Lee B, et al. A case management report: a collaborative perioperative surgical home paradigm and the reduction of total joint arthroplasty readmissions. Perioper Med (Lond). 2016;5:27. [PubMed: 27777752]

19. Zuckerman RB, Joynt Maddox KE, Sheingold SH, Chen LM, Epstein AM. Effect of a Hospitalwide Measure on the Readmissions Reduction Program. New England Journal of Medicine. 2017;377(16):1551-1558. [PubMed: 29045205]

20. Pope GCKJ, Ellis RP, et al. Risk adjustment of Medicare capitation payments using the CMS-HCC model. Health care financing review. 2004;25:119-141. [PubMed: 15493448]

21. Diez Roux AV, Merkin SS, Arnett D et al. Neighborhood of residence and incidence of coronary heart disease. N Engl J Med. 2001;345(2):99-106. [PubMed: 11450679]

22. Saucedo JM, Marecek GS, Wanke TR, Lee J, Stulberg SD, Puri L. Understanding readmission after primary total hip and knee arthroplasty: who's at risk? J Arthroplasty. 2014;29(2):256-260. [PubMed: 23958236]

23. Plate JF, Brown ML, Wohler AD, Seyler TM, Lang JE. Patient Factors and Cost Associated with 90-Day Readmission Following Total Hip Arthroplasty. J Arthroplasty. 2016;31(1):49-52. [PubMed: 26278485]

24. Kurtz SM, Lau EC, Ong KL, Adler EM, Kolisek FR, Manley MT. Hospital, Patient, and Clinical Factors Influence 30- and 90-Day Readmission After Primary Total Hip Arthroplasty. J Arthroplasty. 2016;31(10):2130-2138. [PubMed: 27129760]

25. Moschini M, Gandaglia G, Dell'Oglio P, et al. Incidence and Predictors of 30-Day Readmission in Patients Treated With Radical Cystectomy: A Single Center European Experience. Clinical genitourinary cancer. 2016;14(4):e341-346. [PubMed: 26797584]

26. Borza T, Jacobs BL, Montgomery JS, et al. No Differences in Population-based Readmissions After Open and Robotic-assisted Radical Cystectomy: Implications for Post-discharge Care. Urology. 2017;104:77-83. [PubMed: 28267606]

27. Ricciardi BF, Oi KK, Daines SB, Lee YY, Joseph AD, Westrich GH. Patient and Perioperative Variables Affecting 30-Day Readmission for Surgical Complications After Hip and Knee Arthroplasties: A Matched Cohort Study. J Arthroplasty. 2016.

28. Gani F, Lucas DJ, Kim Y, Schneider EB, Pawlik TM. Understanding Variation in 30-Day Surgical Readmission in the Era of Accountable Care: Effect of the Patient, Surgeon, and Surgical Subspecialties. JAMA Surg. 2015;150(11):1042-1049. [PubMed: 26244543]

29. Packiam VTAV, Pariser JJ, Cohen AJ ea. Redefining the implications of nasogastric tube placement following radical cystectomy in the almivopan era. World journal of urology. 2016.

30. Krishnan N, Liu X, Lavieri MS, et al. A Model to Optimize Followup Care and Reduce Hospital Readmissions after Radical Cystectomy. The Journal of urology. 2016;195(5):1362-1367. [PubMed: 26682758] 


\section{Adjusted readmission rates after radical cystectomy}

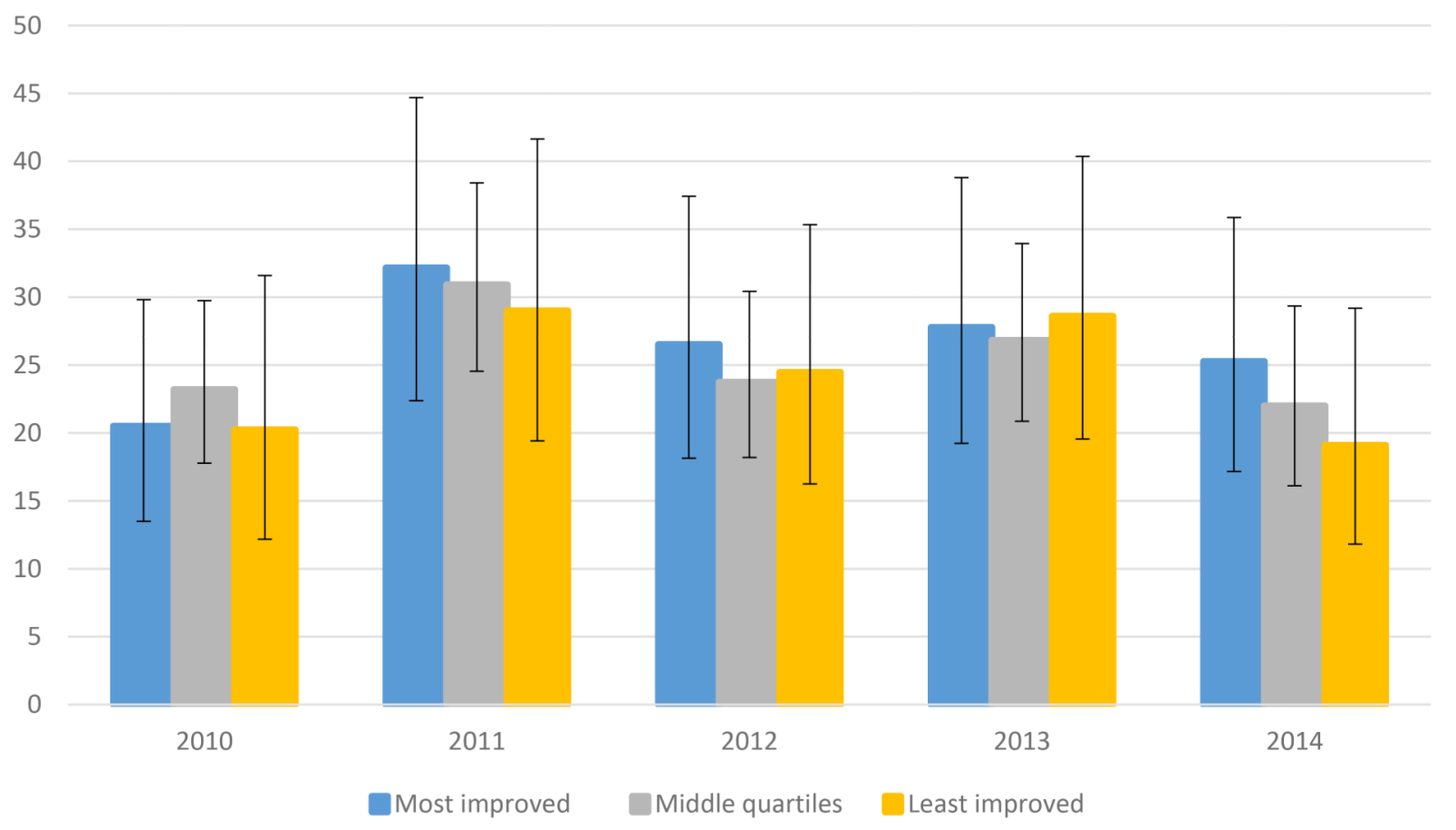

Figure 1.

Patient-level adjusted annual readmission rates after radical cystectomy, stratified by hospital improvement quartile. 


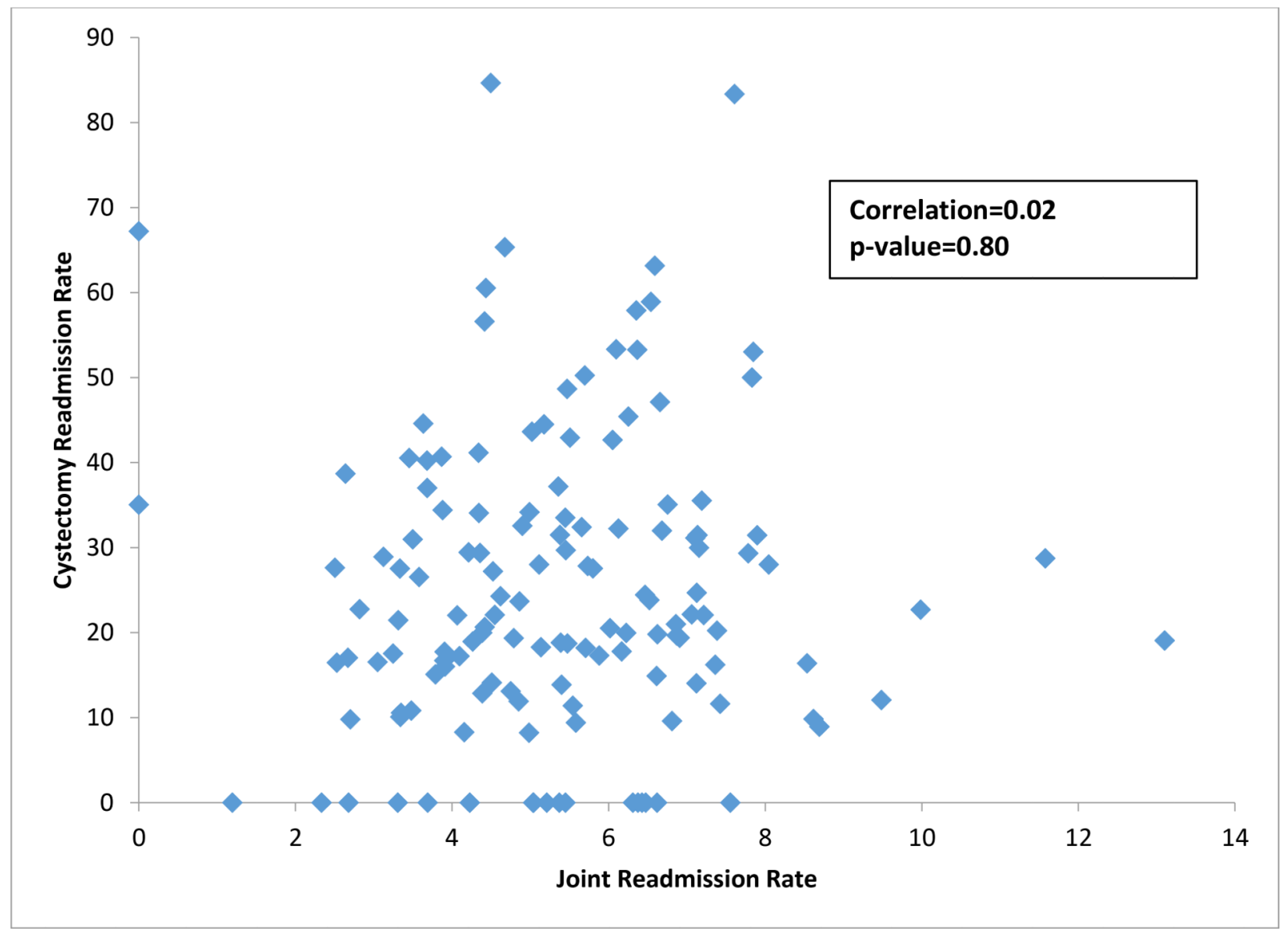

Figure 2.

Scatterplot of a hospital's adjusted radical cystectomy readmission rates against adjusted readmission rates after major joint surgery form hospitals in the top quartile. Analysis limited to hospitals performing at least 5 major joint surgeries and 5 cystectomies annually in our $20 \%$ sample (equivalent to 25 procedures in a $100 \%$ Medicare cohort). 
Table 1.

Comparison of radical cystectomy patient characteristics stratified by the hospital quartile for improvement in major joint surgery readmissions.

\begin{tabular}{|c|c|c|c|c|c|}
\hline \multirow[t]{2}{*}{ Characteristic } & $\begin{array}{c}\text { Total Cystectomy } \\
\text { Cohort }\end{array}$ & $\begin{array}{c}\text { Most improved } \\
\text { quartile }\end{array}$ & Top quartiles & $\begin{array}{c}\text { Least improved } \\
\text { quartile }\end{array}$ & \multirow[t]{2}{*}{ p-value } \\
\hline & $N=2,394$ & $N=606$ & $N=1,266$ & $\mathrm{~N}=522$ & \\
\hline Mean Age (SD) & $75.7(5.8)$ & $75.6(6.0)$ & $75.7(5.8)$ & $75.9(5.8)$ & 0.47 \\
\hline \multicolumn{6}{|l|}{ Race/Ethnicity (\%) } \\
\hline White & 93.1 & 92.2 & 94.2 & 91.6 & 0.68 \\
\hline Black & 4.1 & 4.1 & 3.4 & 5.6 & 0.26 \\
\hline Hispanic & 0.6 & 0.2 & 0.5 & 1.3 & 0.02 \\
\hline Asian/Other & 2.0 & 3.3 & 1.6 & 1.3 & 0.03 \\
\hline Mean Number of HCCs (SD) & $2.0(1.7)$ & $2.0(1.7)$ & $2.0(1.7)$ & $2.1(1.8)$ & 0.61 \\
\hline \multicolumn{6}{|l|}{ Socioeconomic class $(\%)$} \\
\hline Low & 32.2 & 33.2 & 32.3 & 30.8 & 0.40 \\
\hline Medium & 33.1 & 34.8 & 33.4 & 30.5 & 0.12 \\
\hline High & 32.2 & 30.4 & 31.7 & 35.4 & 0.07 \\
\hline \multicolumn{6}{|l|}{ Residential area (\%) } \\
\hline$\geq 1$ million metropolitan county & 42.3 & 38.1 & 40.3 & 52.1 & $<.0001$ \\
\hline$<1$ million metropolitan county & 33.4 & 33.7 & 34.8 & 29.9 & 0.17 \\
\hline$>2,500$ urban population & 21.1 & 24.6 & 21.4 & 16.1 & 0.0004 \\
\hline Rural or $<2,500$ urban population & 3.1 & 3.5 & 3.6 & 1.7 & 0.07 \\
\hline \multicolumn{6}{|l|}{ Hospital Size (\%) } \\
\hline$\underline{2} 50$ beds & 10.7 & 11.2 & 9.3 & 13.2 & 0.31 \\
\hline $251-500$ beds & 29.1 & 23.4 & 30.8 & 31.6 & 0.002 \\
\hline$>500$ beds & 60.2 & 65.3 & 59.9 & 55.2 & 0.001 \\
\hline \multicolumn{6}{|l|}{ Hospital Type (\%) } \\
\hline Teaching & 55.4 & 61.1 & 54.1 & 51.7 & 0.002 \\
\hline Urban & 96.8 & 97.5 & 96.1 & 97.9 & 0.68 \\
\hline For-profit & 7.2 & 3.0 & 7.3 & 12.1 & $<.0001$ \\
\hline Minimally Invasive (\%) & 16.2 & 16.5 & 16.7 & 14.6 & 0.37 \\
\hline
\end{tabular}

HCCs, Hierarchical Condition Categories; SD, standard deviation. 